\title{
Observations of circumstellar disks
}

\section{Philippe Stee}

UMR 6525 H. Fizeau, Université de Nice Sophia Antipolis, Centre National de la Recherche Scientifique, Observatoire de la Côte d'Azur, Avenue Copernic, F-06130 Grasse, France email: philippe.stee@oca.eu

\begin{abstract}
In this review I will present recent results obtained between 2005, which was the last "Be-stars" meeting in Sapporo, and 2010, on the observations of circumstellar disks around active hot stars.
\end{abstract}

Keywords. stars: emission-line, Be, stars: early-type, stars: winds, outflows, stars: rotation, stars: mass loss, stars: imaging, techniques: interferometric, techniques: photometric, techniques: polarimetric, techniques: spectroscopic

\section{Introduction}

The observation of circumstellar disks around active $\mathrm{OB}$ stars is a very active and attractive research field and more than 65 papers with the keywords "observations + disks + Be stars" were found between 2005 and 2010 using the Astrophysics Data System (ADS). These papers were "classified" by myself into various (arbitrary) themes, namely photometry (3 papers), spectroscopy (13), polarimetry (3), interferometry (19), binarity (4), model constraints (8), variability (6), i.e. Non Radial Pulsations, outbursts, one-arm oscillations, etc...) and x-rays (6). Note that interferometric techniques have been really fruitful and seem now to be at a mature age with more results on circumstellar disks coming out from these techniques rather than from spectroscopic observations. I have not included in this review model constraints since this is done by Alex Carciofi in his review. Neither have I presented results on x-rays since this is presented in the contribution by David Cohen, also in these proceedings.

Since the field covered by observations of circumstellar disks is very large, we will try to concentrate on some well identified topics and try to answer only five main questions:

- What is the disk geometry?

- Do we have a disk, a wind or both?

- What are the density and temperature distributions within the disk?

- What are the disk kinematics?

- How does the disk form and dissipate?

We must also stress that disk observations are strongly wavelength and time dependent, with the important role played by amateur astronomer in the spectroscopic and photometric long term following or "alert" mode of some interesting targets, see for instance the BeSS databaset. There is also a large spread of measurements, which may sometimes appear to be inconsistent but since active hot stars are by definition variable it is always difficult to conclude if these differences are linked to an intrinsic source variability or due to a different physical interpretation or simply something that went wrong in the interpretation/simulation. Nevertheless, there is also, and hopefully, some general trends that we will try to describe in the following sections. This paper has the following

$\dagger$ http://basebe.obspm.fr 

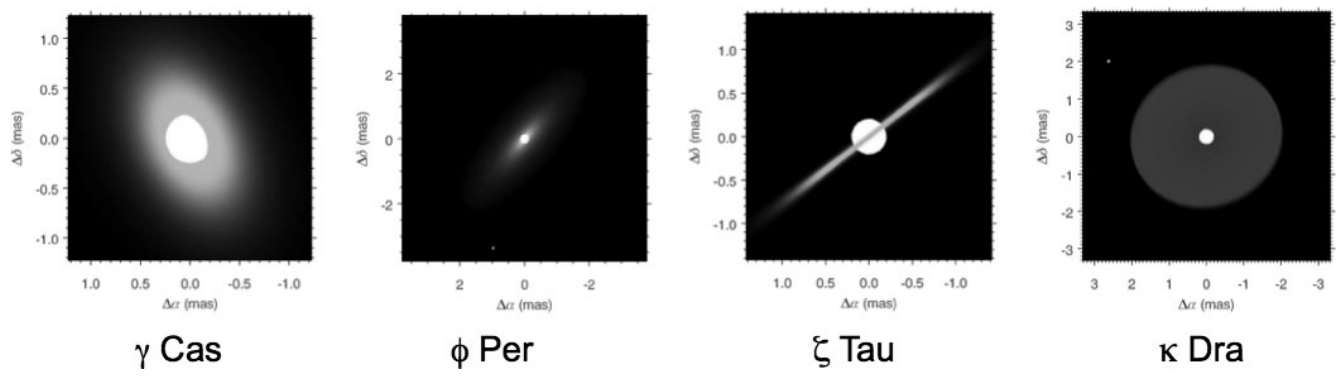

Figure 1. Modeled images using CHARA data by Gies et al. (2007)

structure: in Sect. 2 we present results regarding the extensions of the circumstellar disks using various techniques. In Sect. 3 their shapes and the azimuthal morphology are described. Sect. 4 is dedicated to the determination of their radial density distribution. In Sect. 5 we discuss the recent observations of winds around some Be stars and debate the link between the disk and the wind. Sect. 6 is dedicated to the disk kinematics determination whereas in Sect. 7 we present some possible scenarios regarding the disk formation/dissipation with a special focus on the outburst scenario. The roles of metallicity and binarity are discussed in Sect. 8 and the conclusions, where we will try to answer the initial five questions, are drawn in Sect. 9.

\section{Disk geometry: extension}

Using spectro-astrometry measurements at $1.28 \mu \mathrm{m}$ Oudjmaijer et al. (2008) found an extension of a few dozen stellar radii for $\alpha \mathrm{Col}$ and $\zeta$ Tau whereas Arias et al. (2006) obtained $2.0 \pm 0.8 \mathrm{R}_{\star}$ in Fe II optically thick lines between $4230-7712 \AA$ using the SAC method. The size of the hot CO (2-0 and 3-1 bands of the CO overtone) around the (young) Be star 51 Oph was resolved with VLTI/AMBER at $2.3 \mu \mathrm{m}$ with a size of $0.15 \mathrm{AU}$ and a more distant nearby continuum of $\sim 0.25$ AU by Tatulli et al. (2008) confirming that the $\mathrm{CO}$ band-heads originate in a dust free hot gaseous disk and the continuum emitting region is closer to the star than the dust sublimation radius by at least a factor two. Meilland et al. (2009) have observed 7 classical Be stars with the VLTI/MIDI between 8 and $12 \mu \mathrm{m}$, namely $\mathrm{P}$ Car, $\zeta$ Tau, $\kappa \mathrm{CMa}, \alpha \mathrm{Col}, \delta \mathrm{Cen}, \beta \mathrm{CMi}$, $\alpha$ Arae. They found that the sizes of the disks do not vary strongly with wavelength within this spectral domain which is a very different conclusion compared to B[e] stars, with increasing sizes as a function of wavelength. Moreover the size of $\alpha$ Arae's disk was found to be identical at 2, 8 and $12 \mu \mathrm{m}$, which might be due to disk truncation by a companion. Finally it seems from their studies that envelopes of late type Be stars might be smaller than for early type.

$\gamma$ Cas and $\psi$ Per were observed by Tycner et al. (2006) with the NPOI interferometer in the $\mathrm{H} \alpha$ domain. They found that a uniform disk or a ring-like model were inconsistent with their data and that a Gaussian model was fitting pretty well the measurements. The $\gamma$ Cas disk was also consistent with the orbital parameters already published. Nevertheless, higher precision binary solutions were mandatory to test for a possible disk truncation by the secondary. The disk of $\psi$ Per was found to be truncated by the presence of a companion as already predicted by Waters (1986). $\gamma$ Cas, $\psi$ Per, $\zeta$ Tau and $\kappa$ Dra were observed with the CHARA interferometer in the K-band by Gies et al. (2007) (see Fig. 1). They found, using Gaussian elliptical fits of visibilities, that the disk size 


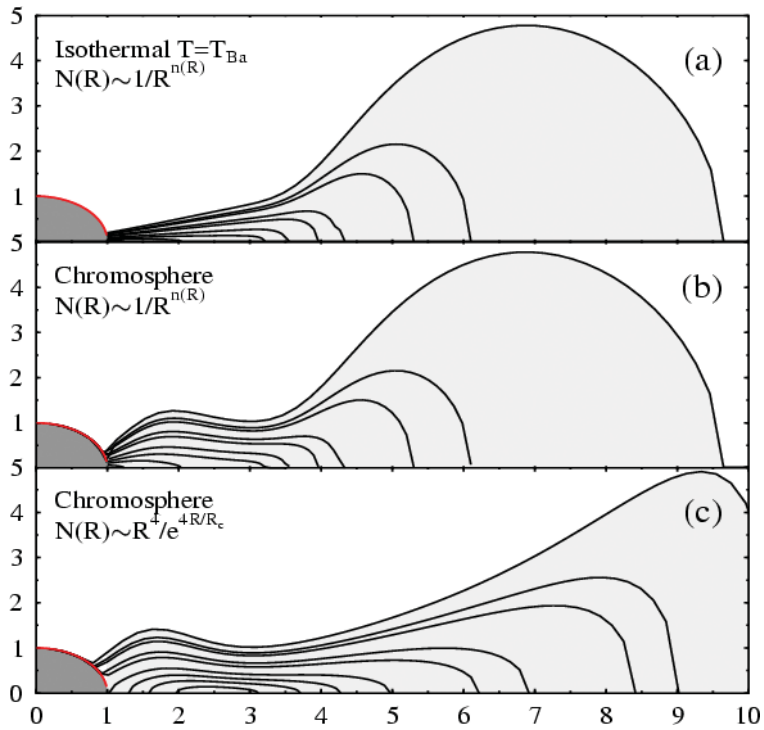

Figure 2. Shape of discs with Keplerian scale heights from Zorec et al. (2007)

in the K-band was smaller than in $\mathrm{H} \alpha$ due to a larger $\mathrm{H} \alpha$ opacity and relatively larger neutral hydrogen fraction with increasing disk radius. All these Be stars are known binaries and this binarity effect was found to be more important for $\psi$ Per and $\kappa$ Dra. Tycner et al. (2008) using the NPOI interferometer observed $\chi$ Oph and obtained a good fit of the $\mathrm{H} \alpha$ emitting disk with a circularly symmetric Gaussian with a FWHM diameter of $3.46 \pm 0.07$ mas. The extension of 48 Per and $\psi$ Per in the $\mathrm{H} \alpha$ domain was determined by Delaa et al. (2010) using a Gaussian disk model and found to be respectively $2.1 \pm$ 0.2 and $4.0 \pm 0.2$ with a flattening ratio of 1.3 and 2.9 .

\section{Disk geometry: shape and the azimuthal morphology}

The shape of the disk around Be stars is still a very active and controversial topic, i.e. a very flattened disk of $1-3^{\circ}$ of opening angle versus more "spheroidal" geometry. Arias et al. (2006) have observed in the Balmer lines 18 southern B stars and found a flattened geometry but without a quantitative value, whereas Zorec et al. (2007) obtained a semiheight scales perpendicular to the equatorial plane $h \leqslant 0.5 \mathrm{R}_{\star}$ from Fe II optically thick line observations of 17 Be stars using the SAC method.

$\zeta$ Tau was found to exhibit asymmetrical IR emission lines (He I, O I, Fe II) and Brackett, Paschen, Pfund series lines with opposite $\mathrm{V} / \mathrm{R}(\mathrm{V}>\mathrm{R})$ compared to $\mathrm{H} \alpha$ Balmer lines $(\mathrm{V}<\mathrm{R})$. This was interpreted by Wisniewski et al. (2007) as a direct evidence of a density wave with different average azimuthal morphology in the inner versus outer disk region. This asymmetry was also measured by Schaefer et al. (2010) with the CHARA/MIRC interferometer in the $\mathrm{H}$ band. They have evidenced this asymmetry along the minor axis of the disk with a $10^{\circ}$ change in the Position Angle (PA) of the disk between 2007, Nov and 2008, Sep. The cyclic variability of the disk of $\zeta$ Tau was successfully explained with a 2D global disk oscillation model by Stefl et al. 2009 and Carciofi et al. 2009 (see Fig. 3) where they have used the NLTE 3D code HDUST to fit simultaneously VLTI/AMBER data and $\mathrm{V} / \mathrm{R}$ variations in the $\mathrm{H} \alpha$ and $\mathrm{Br} \gamma$ lines. An asymmetry was also detected in the disk of $\kappa$ CMa with the VLTI/AMBER interferometer in the K band by Meilland et al. (2007b) but this asymmetry was found to be poorly explained within the "one-armed" 

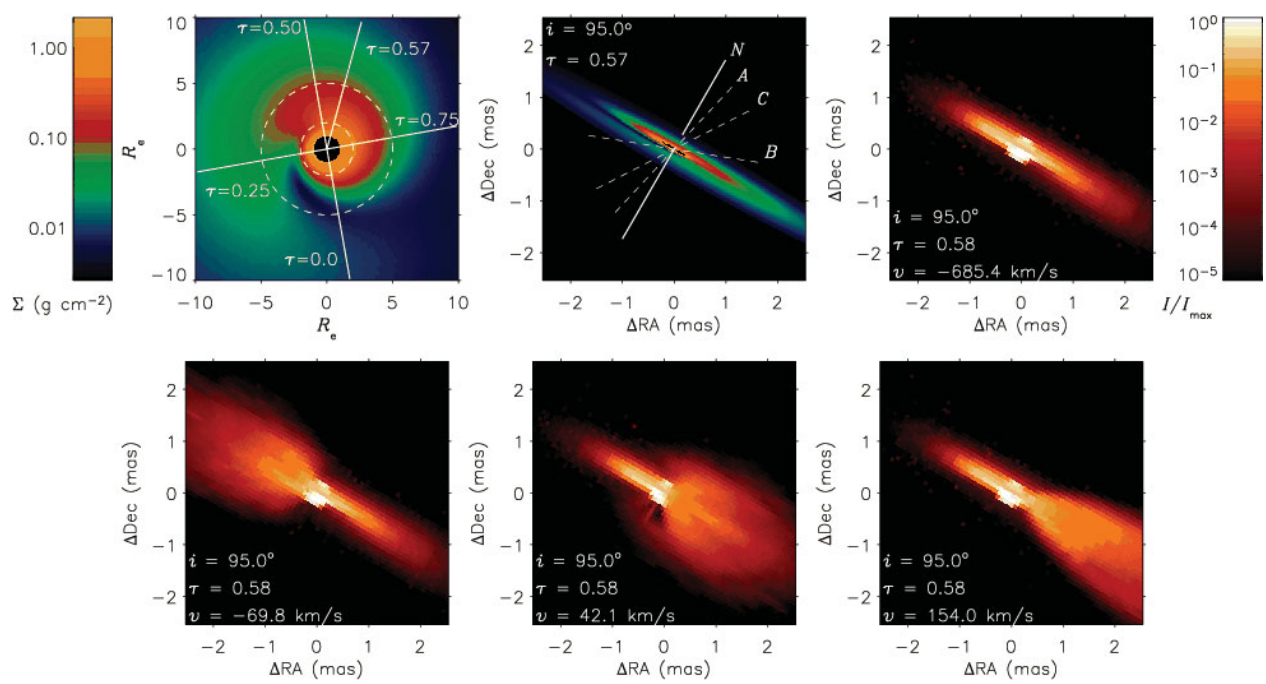

Figure 3. $\zeta$ Tau density perturbation pattern from Carciofi's model (Carciofi et al. 2009)

viscous disk framework Okazaki (1997) since the "pseudo period" they obtained was too long compared to predictions for a disk with a radius of $23 R_{\star}$.

\section{Disk geometry: radial density distribution}

The value of the " $n$ " index of the density distribution within the circumstellar disk is also highly debated in the community. We recall that the density distribution is often described as:

$$
\rho(r)=\rho_{0}\left(\frac{R_{\star}}{r}\right)^{n}
$$

where $R_{\star}$ is the stellar radius and $\rho_{0}$ the density at the base of the disk.

Zorec et al. (2007) found that $n \leqslant 1$ for $\mathrm{r} \leqslant 3 \mathrm{R}_{\star}$, using the SAC method and and a non-LTE interpretation of the FeII line formation in the disc, $\mathrm{n} \sim 0.5$ near the star from near-UV spectropolarimetry measurements (see Fig. 2) whereas Gies et al. (2007) obtained values for $n$ between 0.16 and 3.19 and emphasized that $n$ is smaller in binaries with smaller semi-major axis, namely $\psi$ Per and $\kappa$ Dra, which is a confirmation that binary companions do influence the disk properties. Interestingly, Jones et al. (2008) have fitted observed $\mathrm{H} \alpha$ visibilities from the NPOI interferometer and observed $\mathrm{H} \alpha$ line profiles using the NLTE code BEDISK. They obtained $n$ values of $4.2,2.1,4.0$ and $\rho_{0}=$ $1.510^{-10}, 3.010^{-12}$ and $8.010^{-10} \mathrm{~g} \mathrm{~cm}^{-3}$ respectively for $\kappa$ Dra, $\beta$ Psc, $v$ Cyg. Note that there is a large discrepancy between the value found by Gies et al. (2007), i.e. 0.16 and the one from Jones et al. (2008), i.e. 4.2. This discrepancy is deeply discussed in

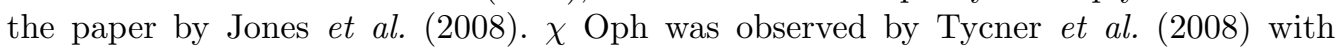
the NPOI interferometer and modeled with BEDISK. They obtained $n=2.5$ and $\rho_{0}=$ $2.010^{-11} \mathrm{~g} \mathrm{~cm}^{-3}$.

The Be star $\delta$ Sco was also intensively observed since this binary star is supposed to have its companion at periastron around July 2011(some American colleagues would guess July 4th, I bet on July 14 !). Thus, Carciofi et al. (2006) have studied its disk properties from continuum modeling, photometric and spectro-polarimetric observations. They found that in order to explain their observations (between 2000 and 2005) they have to advocate for a change in the mass loss rate AND in the disk geometry. The disk was 


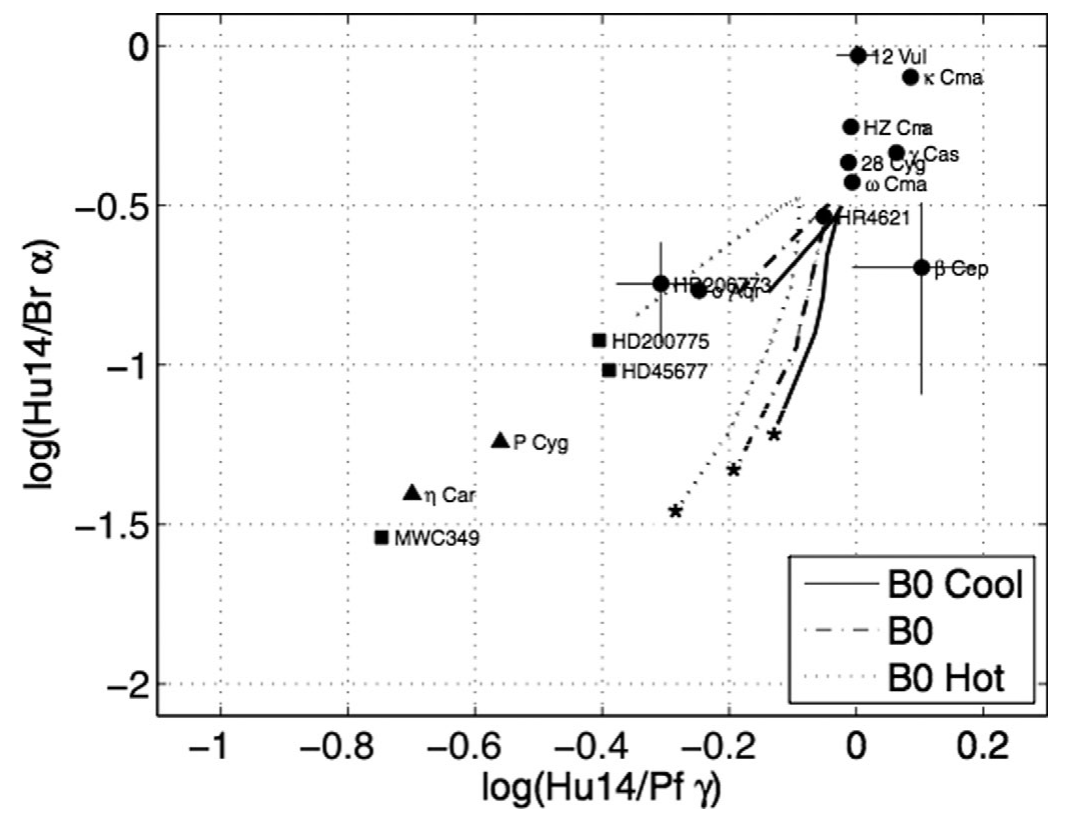

Figure 4. Predicted line flux ratios computed from theoretical disc models by Jones et al. (2009) for values of $n=3.0,3.5$ and 4.2 with a range of $\rho_{0}$

found to be non isothermal and fully ionized. The inner disk is supposed to be unflared in contrast to large flaring expected for isothermal models. They also found that $n \neq 3.5$ as this is the case for isothermal disks and they obtained a total mass loss of $1.510^{-9} \mathrm{M}_{\odot}$ $\mathrm{yr}^{-1}$ and a disk radius of $7 \mathrm{R}_{\star}$. New photometric observations of $\delta$ Sco were carried out in 2006 (and taking into account the 10 months observations earlier by Carciofi) in the J, $\mathrm{H}$ and $\mathrm{K}$ bands by Halonen et al. (2008). From the modeling of the $\mathrm{H} \alpha$ emiision line they obtained $\rho_{0}=5.010^{-10} \mathrm{~g} \mathrm{~cm}^{-3}$ and $n=4.0$ even if they mention that a range for $n$ from 3.5 to 4.5 and $\rho_{0}$ from $5.010^{-11}$ to $5.010^{-10}$ is acceptable. They found a "global" agreement between Carciofi's code and BEDISK and a detailed comparison between both codes is foreseen. On the other hand, we recall that Zorec et al. (2007) obtain $n<1$ for $\mathrm{r}<3 \mathrm{R}_{\star}$ and that the density distribution near the star is constant or increasing. This $n<$ value is also important in order to reproduce the "flat" distribution of the linear polarization observed in the far-UV. Finally Carciofi et al. (2006) explain that in order to reproduce the variations in brightness of the system, some material must be ejected at higher stellar latitudes.

From the Spectral Energy Distribution (SED) study, using spectro-photometric data from 0.4 to $4.2 \mu \mathrm{m}$ of northern sky Be stars Touhami et al. (2010), found that stars with a strong $\mathrm{H} \alpha$ excess have also an IR exces. This IR excess correlates with the $\mathrm{H} \alpha$ Equivalent Width (EW), with the largest scatter from the data for densest and largest disks. They obtain a better correlation with high excitation transitions, for instance Hu14. The IR continuum and these high excitation transitions are supposed to occured in the inner and dense part of the disk. Thus, the less marked correlation between the IR excess and the $\mathrm{H} \alpha \mathrm{EW}$ might be linked with changes in the density distribution in the outer part of the disk, maybe due to a temporal evolution of the disk or the influence of a binary companion. The IR domain is a useful spectral domain to constrain the disk geometry. For instance Jones et al. (2009) used the observed flux ratio Hu14/Br $\gamma$ and $\mathrm{Hu} 14 / \mathrm{Pf} \gamma$ defined by Lenorzer et al. (2002) to constrain basic properties of Be disks with 


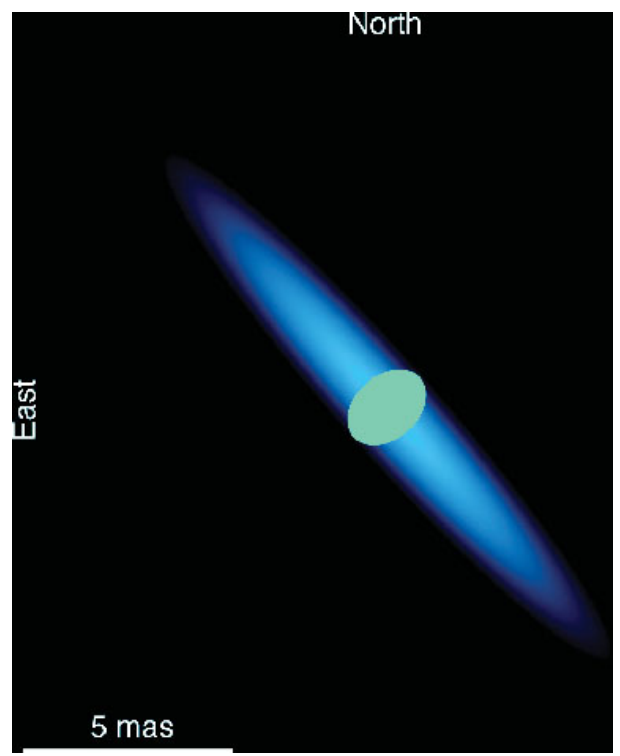

Figure 5. Graphical representation of the best-fit model intensity distribution of Achernar by Kervella \& Domiciano (2006)

the BEDISK code (see Fig. 4). They observed a continuum turn-down at $\mathrm{mm}$ and radio wavelengths which was explained by a geometrical disk change, i.e. an increase of $n$ at large radial distances from the star, already predicted by Waters et al. (1991).

\section{Disk geometry: disk and/or wind ?}

Recently polar winds along the rotational axis of Be disks were evidenced using interferometric techniques. For instance Kervella \& Domiciano (2006) have measured a polar extension on the fast rotating Be star Achernar using the VLTI/VINCI instrument at 1.631 and $2.175 \mu \mathrm{m}$ (Fig. 5). This wind have an extension of $17.6 \pm 4.9$ mas and a relative (envelope to star) near-IR flux contribution of $4.7 \pm 0.3 \%$. Kanaan et al. (2008) used a wind+disk model to interpret the whole set of VLTI/VINCI data of Achernar in the K band and found the these data cannot be explained by a rotationally distorted Be star with a companion alone. A polar wind contribution is mandatory to fit the data. This polar wind contribution, interpreted as a polar wind, does not appear to be linked to the presence of a disk or a ring. Thus, it seems possible to have a wind without a disk in the circumstellar environment of a Be star. Meilland et al. (2007a) have also evidenced a dense equatorial (Keplerian) disk with a polar wind along the rotational axis of $\alpha$ Arae using the VLTI/AMBER instrument in the K band (Fig. 6).

Another interesting possibility is to test if the disk formation/dissipation is creating rings around the central star or not. Using theoretical SED, Br $\gamma$ line profiles and visibilities for two scenarios explaining the disk dissipation: an outburst scenario forming rings or a slowly decreasing mass loss, Meilland et al. (2006) have shown that a clear signature of the ring dissipation scenario would be the disappearance of the high velocity tails in the emission lines and a nearly constant peak separation, as well as a clear interferometric signature with an increasing second lobe and a displacement of the first zero of the visibility. Note that a ring like structure was already evidenced by Millour et al. (2011) 


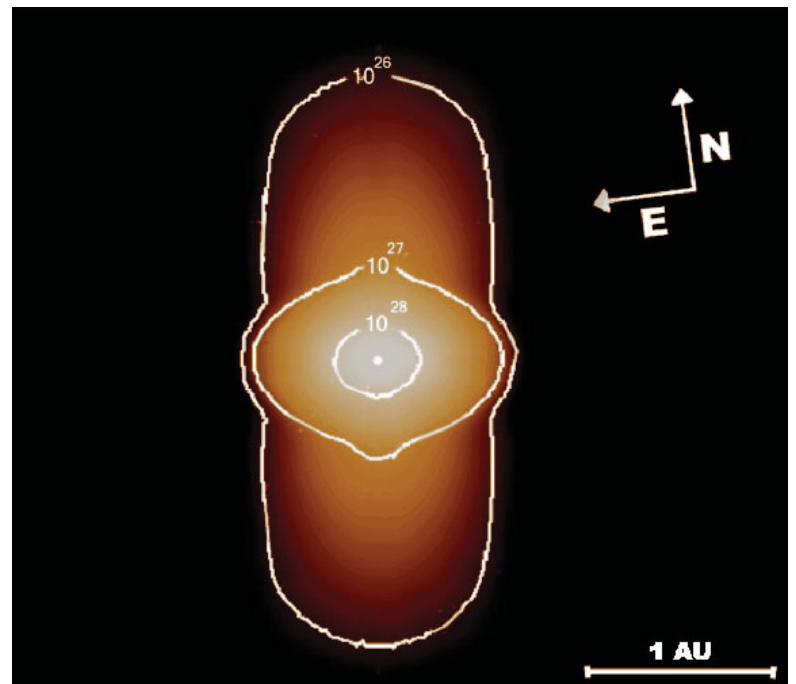

Figure 6. Intensity map in the continuum at $2.15 \mathrm{~m}$ obtained with SIMECA from Meilland et al. (2007a) best model parameters

around the $\mathrm{B}[\mathrm{e}]$ star HD 62623 from a direct image reconstruction method without any a priori model from VLTI/AMBER spectrally resolved data.

\section{Circumstellar disks: kinematics}

Thanks to spectrally resolved interferometric observations, direct measurement of the kinematics with the disk of Be stars was possible. Meilland et al. (2007a) was the first to directly determine the Keplerian rotation of the disk around $\alpha$ Arae with the VLTI/ AMBER instrument in the $\mathrm{K}$ band. On the other side, same type of measurements were applied for $\kappa$ CMa by Meilland et al. (2007b) who obtained that the rotation within the disk was not Keplerian and $\beta=0.32$ was found for this star assuming that the rotation law follows $\mathrm{V}_{\phi}(\mathrm{r}, \theta)=\sin \theta\left(\frac{R}{r}\right)^{\beta}$. The kinematics within the disk of the $\mathrm{B}[\mathrm{e}]$ star HD 62623 was also found to be Keplerian by Millour et al. (2011) and the first spectrally resolved reconstructed images were obtained, again without any a priori model from VLTI/AMBER measurements. A comparison between $\alpha$ Arae, $\kappa$ CMa and Achernar kinematics was presented by Stee \& Meilland (2009) and summarized in Table 1.

48 Per and $\psi$ Per were also observed by Delaa et al. (2010) with the CHARA/VEGA instrument at $0.65 \mu \mathrm{m}$ and the rotation within the disk of 48 Per was found to be Keplerian, i.e. $\beta=0.5$ whereas for $\psi$ Per a $\beta=0.35$ value was obtained which is very similar to the one obtained for $\kappa$ CMa, i.e. $\beta=0.3$. Thus, the departure of the Keplerian rotation might be linked to a density perturbation such as a "one-armed" oscillation and/or a perturbation due to a close enough companion. Note that Keplerian rotation was also found for $\beta$ CMi's disk by Oudmaijer et al. using very high resolution spectroscopic data (see his poster contribution in these proceedings).

Another important parameter to obtain (also needed to constrain the disk kinematics) is the inclination angle of the rotational axis with respect to the line of sight. For that purpose, Mackay et al. (2009) have computed theoretical normalized stokes visibilities of Be disks and has shown that if we are able to obtain an accuracy on the interferometric polarization observations better than $10^{-3}-10^{-4}$, Stokes Q visibilities can remove model degeneracies and can estimate the disk inclination angle. 


\begin{tabular}{|c|c|c|c|}
\hline \multicolumn{4}{|c|}{ Equatorial Disk } \\
\hline & $\alpha$ Arae & $\kappa \mathrm{CMa}$ & Achernar \\
\hline $\begin{array}{l}\text { Stellar } \\
\text { Rotation }\end{array}$ & $97 \% \mathrm{~V}_{c}$ & $52 \% \mathrm{~V}_{c}$ & $\sim \mathrm{V}_{c}$ \\
\hline $\begin{array}{l}\text { Radius } \\
\text { (K band) }\end{array}$ & $32 \mathrm{R}_{\star}$ & $\begin{array}{c}23 \mathrm{R}_{\star} \\
\text { independent of } \lambda\end{array}$ & $\begin{array}{c}\text { Intermittent } \\
\mathrm{R}_{\max } \sim 4.8 \mathrm{R}_{\star} \text { if Keplerian }\end{array}$ \\
\hline $\begin{array}{c}\text { Flux } \\
(\mathrm{K} \text { band })\end{array}$ & $40 \%$ & $50 \%$ & $<5 \%(2002)$ \\
\hline Expansion & negligible & negligible & $0.2 \mathrm{kms}^{-1}$ \\
\hline $\begin{array}{l}\text { Disk rotation } \\
(\beta \text { parameter })\end{array}$ & $\begin{array}{c}0.48 \\
\text { (quasi Keplerian) }\end{array}$ & $\begin{array}{c}0.3 \\
\text { (sub Keplerian) }\end{array}$ & $\begin{array}{l}\text { no disk detected } \\
\text { in } 2000\end{array}$ \\
\hline \multicolumn{4}{|c|}{ Polar Wind } \\
\hline Extension & $>10 \mathrm{R}_{\star}$ & not detected & $>10 \mathrm{R}_{\star}$ \\
\hline $\begin{array}{c}\text { Flux } \\
(\text { K band })\end{array}$ & $1-5 \%$ & $\mathrm{X}$ & $\begin{array}{l}3-4 \%(2002) \\
\text { (H band) }\end{array}$ \\
\hline Opening angle & $50 \pm 10^{\circ}$ & $\mathrm{X}$ & $5-40^{\circ}$ \\
\hline
\end{tabular}

Table 1. Comparison of the stellar rotation, geometry and kinematics of the circumstellar disk of the 3 studied Be stars $\alpha$ Arae, $\kappa$ CMa from Stee \& Meilland (2009).

\section{Disk formation/dissipation: Non Radial Pulsations (NRP) and Outbursts}

Observational proof for a direct link between NRP or Outbursts and disk formation is growing. For instance, using multiple epochs $\mathrm{H} \alpha$ spectroscopy of 47 stars in the open cluster NGC 3766 Mc Swain et al. (2008) have confirmed that 16 objects were Be stars and discovered one new Be star. No particular stage of main sequence evolution were found but they are all rapid rotators (with rotation at least between $70-80 \%$ of the critical velocity). The have also observed disk size changes which are consistent with $\operatorname{NRP}(l=2, \mathrm{~m}= \pm 2)$ for the formation of these highly variable disks. Note that, in this very interesting paper, they were also able to estimate the disk formation rates for many targets. Mennickent et al. (2009) have done L-band observations of 13 outbursting Be stars with the ISAAC instrument. They found different groups of stars regarding the $\operatorname{Br} \alpha, \operatorname{Pf} \gamma$ and Humphreys lines optical depth. This was consistent with the description by de Wit (2006) for Be stars outbursts in termes of ejection of an optically thick disk that expands and becomes optically thin before dissipation. A large broadening was also observed in the IR lines which was attributed to vertical velocity fields near the central star. The outburst scenario was also investigate by Kanaan et al. (2008) and was found to be compatible to interpret spectroscopic observations of Achernar and the formation of an outflowing ring with a radial velocity $\sim 0.2 \mathrm{kms}^{-1}$. Rapid polarization variability of Achernar was also interpreted as evidence of photospheric and circumstellar activity by Carciofi et al. (2007). Thanks to a detailed modeling of the polarization they suggest that short term variations originate from discrete mass ejection events which produce transient inhomogeneities in the inner disk. They also explain the observed long term variations as due to the formation of an inner ring following one or several mass ejection events. The same kind of study was done by Wisniewski et al. (2010) using spectro-polarimetric observations to study the formation/dissipation of the disk around $\pi$ Aquarii and 60 


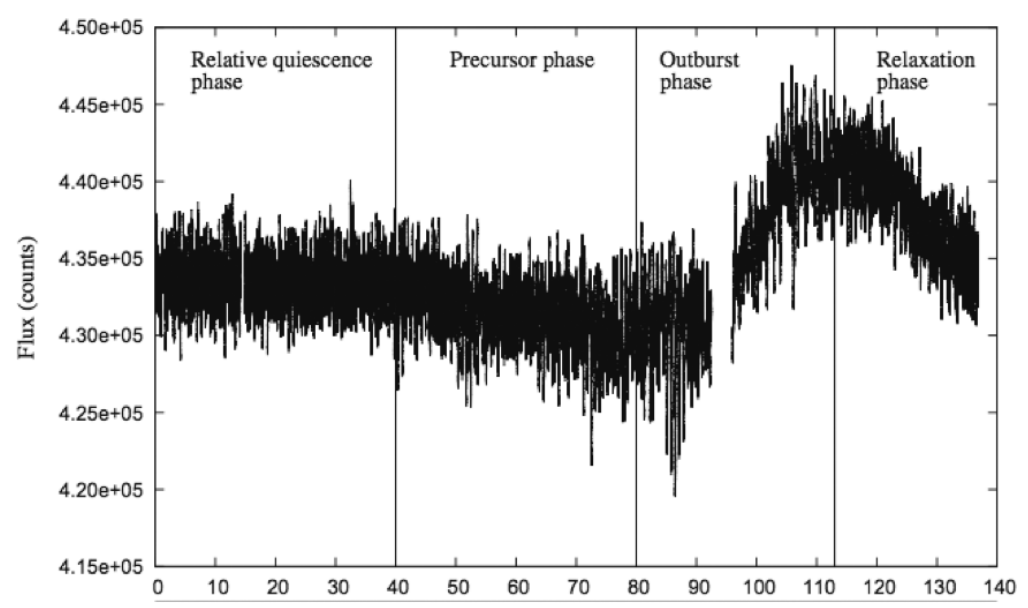

Figure 7. CoRoT light curve of HD 49330 in flux from Huat et al. (2009)

Cygni. They observed a minor outburst during a quiescent phase, with similar lifetimes as for $\mu$ Cen, which was interpreted as injection and viscous dissipation of individual blobs into the inner disk. Observed deviations from the mean intrinsic polarization angle during polarization outburst may be due to deviations from axisymmetry. These deviations are certainly indicative of injection and circularization of new blobs into the inner disk, in the plane of the disk or in a slightly inclined (non coplanar) orbit.

Nevertheless, the first clear and direct evidence of an ejection of matter that coincides with the constructive beating of two main frequencies during an outburst phase was obtained by Huat et al. (2009) and Floquet et al. (2009) from the observation of the CoRoT light curve of HD 49330 (see Fig. 7). What is still not clear is the fact that the variations in pulsation modes are producing the outburst, as already predicted by Rivinius et al. (1998) or if this is the occurence of the outburst that is producing the excitation of modes, e.g. a change in the stellar structure. Monitoring the precursor phase of the outburst of $28 \mathrm{CMa}$ (October 2008) in photometry, high resolution optical and near IR spectroscopy and interferometry, Stefl et al. (2010) have observed cyclic smaller outbursts. These observations seem to be in better agreement with the dynamical model by Okazaki (2007) where the inner disk has (partially) reacreted to the central star.

Regarding possible disk formation/dissipation scenarios, Granada et al. (2010) have observed 8 Be stars in the $\mathrm{K}$ and $\mathrm{L}$ bands with the GEMINI/NIRI instrument. Two distinct groups were found: the first one with optically thin IR lines (28 Tau, 66 Oph, V923 Aql, $28 \mathrm{Cyg}$ ) and the second one with optically thin IR lines (88 Her, BK Cam) and one Be star in between: EW Lac. For $28 \mathrm{Tau}, 66 \mathrm{Oph}$ and $28 \mathrm{Cyg}$ the $\mathrm{H} \alpha$ weakening observed is consistent with a dissipating disk. For 88 Her and BK Cam the disk seems to be growing certainly due to recent mass ejection episodes. Observed variations in $\mathrm{H} \alpha$ and polarization were interpreted as direct evidence of the spatial motion of the disk and the presence of a precessing disk outside the equatorial plane. The innermost regions of the envelope were found to rotate faster and/or scattering effects must be relevant in these dense regions.

Since many Be stars seem to have permanent disks whereas some others have very variable disks we may wonder if this is due to different physical properties. For that purpose, Mc Swain et al. (2009) have studied the disk formation/dissipation in 4 southern open clusters. 296 stars were observed at multiple epochs in $\mathrm{H} \alpha$. They have detected 12 new transients whereas 17 additional Be stars with relatively stable disks were confirmed. 
Thus, they have investigated physical differences between these transient Be stars, stable Be and normal B type stars. Be stars were found to be all faster rotators but no significant physical differences between transient and stable Be stars were evidenced.

\section{Disk formation: metallicity, critical rotation and binarity}

Metallicity has certainly a great effect on the evolution and the formation of Be star disks as demonstrated by Martayan et al. (2010) using $\mathrm{H} \alpha$ slitless spectroscopy of 4437 stars with a survey complete to B2/B3 spectral types on the main sequence. They obtained that in the Small Magellanic Cloud (SMC) Be stars are more frequentby a factor 3-5 and that the fractional critical angular rotation rate $\Omega / \Omega_{c}$ is the main parameter governing the occurence of the Be phenomenon. This Be phenomenon seems to cover preferentially the second half of the main sequence evolution. Moreover, considering the evolution, the initial mass and the metallicity effect, $\Omega / \Omega_{c}$ is still the main dominant parameter.

Binarity was also very often advocate as THE clue for the Be phenomenon, i.e. the formation of a circumstellar disk in a close binary system. Even if the number of binary Be stars is rapidly growing, especially with recent interferometric detections, and if binarity is not the main physical process to form a Be disk, it has certainly very important effects on the disk size and evolution as we will see in the following examples. For instance Catanzaro (2008) has observed $\beta$ Cephei, even if we may wonder if $\beta$ Cephei is really a typical Be star, nevertheless its $\mathrm{H} \alpha$ emission line seems to be typical for Be stars. In fact, this emission is attributed to the secondary component since $\beta$ Cephei is a spectroscopic binary system. From archived spectra he has recovered the evolution of the $\mathrm{H} \alpha$ line profile od the secondary as a function of time. He found that the typical double-peak emission was strongly variable and was able to estimate the vsini of the secondary to be $230 \mathrm{kms}^{-1}$ and estimate the outer radius of the disk to be $3.28 \mathrm{R}_{\star}$ (assuming a Keplerian rotation). He also found thaht the Radial Velocity (RV) and V/R variations observed were in agreement with Okazaki (1996) model. Achernar was also classified as "binary" since the detection of a companion at a few tens of AU from VLTI/VISIR measurements by Kervella \& Domiciano (2007). The secondary was found to be compatible with a late A type main sequence star with a Position Angle (PA) almost aligned with the equatorial plane of Achernar. The projected linear separation they obtain was 12.3 AU. Last but not least, $\delta$ Cen was also detected as a new binary by Meilland et al. (2008) using VLTI/AMBER spectro interferometry in the $\mathrm{H}$ and $\mathrm{K}$ bands. Assuming a radius of 5.9 $\mathrm{R}_{\odot}$, a distance of $121 \mathrm{pc}$, the measured separation of 68.7 mas they obtained corresponds to $300 \pm 50 \mathrm{R}_{\star}$. The companion spectral class ranges between B4V and A0III, its mass between $4-7 \mathrm{M}_{\odot}$ and the Period lower limit estimates was equal to 4.6 years.

\section{Conclusion}

Finally we may wonder if it is possible to answer to the 5 previous questions ?

- What is the disk geometry?

A few mas (a few tens of $R_{\star}$ ), thin close to the central star but flaring after a few stellar radii. Truncated when the Be star is embedded within a binary system. The opening angle of the disk is still poorly constrained due mainly to the disk inclination uncertainty. Nevertheless, it is certainly NOT as thin as it was previously supposed, i.e. with a $1-2^{\circ}$ of opening angle. The disk geometry, as seen during this review, is strongly wavelength (or chemical species) dependent. 
- Do we have a disk, a wind or both ?

Certainly both with the possibility to have a wind but no disk. They were strong debates during this IAU meeting regarding the wind detection by interferometric measurements and the fact that this detection was model dependent or not. There is clearly a polar extension in the interferometric data. Is it a wind or something else, the answer is not trivial and the solution may not be unique. On the other side, these interferometric observations are compatible with a polar wind model, which include a NLTE radiative transfer simulation, free-free and free-bound continuum emission. Whether this wind as to be optically thick or not is not, from my point of view, the right question: what we only need is some photons coming out of this wind and reaching the detector regardless the optical thickness of the wind. Moreover, Achernar's wind was observed in the near-IR continuum (K-band) and, if this continuum was optically thick, it would infer that the Balmer lines are also (and even more) optically thick. Since Achernar is seen close to edge-on we would have seen a strong "single-peak" $\mathrm{H} \alpha$ emission line which is not the case since the $\mathrm{H} \alpha$ line at this epoch was very faint and "double-peaked". Does it means that the wind opacity is sufficient to be seen in the near-IR continuum and that the $\mathrm{H} \alpha$ source function is too faint due to a very strong Hydrogen ionization caused by the hotter polar caps ? If the wind is optically thick in the $\mathrm{K}$ band it must be very closed to the star, i.e. $\leqslant 1 R_{\star}$ and optically thin and extended further away. A nice confirmation of the stellar wind hypothesis would be to observer other Bn stars and try to see with VLTI/AMBER or VEGA/CHARA if there is also an extended emission detected along their polar axis, mainly due to the Von Zeipel effect.

- How is the density and temperature distribution within the disk? Well, this is not very easy to answer. Clearly the $n$ index is $\neq 3.5$ but can change as a function of the stellar radius. The Temperature seems to be cooler in the disk mid-plane and not isothermal. In many cases, this density distribution seems not to be axisymmetric with nice confirmations of the $2 \mathrm{D}$ global disk oscillations scenario.

- What is the disk kinematics?

Clearly Keplerian but with some departure. Are these departure linked to these global disk oscillations in some cases or related to a putative companion? The situation for these non Keplerian detections are not very clear and have certainly to be confirmed. Whatever this rotation is, the disk around Be star is clearly dominated by the rotation with only a very small radial velocity $\left(<1 \mathrm{kms}^{-1}\right)$.

- How do the disk form and dissipate?

The critical rotation and the critical angular rotation rate $\Omega / \Omega_{c}$ together with the outbursts scenario are clearly the most favorable physical processes to create a circumstellar disk.

\section{Acknowledgements}

This work has been supported by the French Program National en Physique Stellaire (PNPS) and by the Centre National de la Recherche Scientifique (CNRS). This research has made use of SIMBAD database, operated at CDS, Strasbourg, France and of NASA's Astrophysics Data System.

\section{References}

Arias, M. L., Zorec, J., Cidale, L., Ringuelet, A. E. et al. 2006, A\& A, 460, 821

Catanzaro, G. 2008, MNRAS, 387, 759

Carciofi, A. C., Miroshnichenko, A. S., Kusakin, A. V., Bjorkman, J. E. et al. 2006, ApJ, 652, 1617 
Carciofi, A. C., Magalhães, A. M., Leister, N. V., Bjorkman, J. E. et al. 2007, ApJ (Letters), $671, \mathrm{~L} 49$

Carciofi, A. C., Okazaki, A. T., Le Bouquin, J.-B., Štefl, S. et al. 2009, A\&A, 504, 915

Delaa, O., Stee, P., Meilland, A. et al. 2010, A\&A, in preparation

de Wit, W. J., Lamers, H. J. G. L. M., Marquette, J. B. \& Beaulieu, J. P. 2006, A\& A, 456, 1027

Floquet, M., Hubert, A.-M., Huat, A.-L., Frémat, Y. et al. 2009, A\&A, 506, 103

Gies, D. R., Bagnuolo, Jr., W. G., Baines, E. K., ten Brummelaar, T. A. et al. 2007, ApJ, 654, 527

Granada, A., Arias, M. L. \& Cidale, L. S. 2010, AJ, 139, 1983

Halonen, R. J., Jones, C. E., Sigut, T. A. A., Zavala, R. T. et al. 2008, PASP, 120, 498

Huat, A.-L., Hubert, A.-M., Baudin, F., Floquet, M. et al. 2009, A\&A, 506, 95

Jones, C. E., Molak, A., Sigut, T. A. A., de Koter, A. et al. 2009, MNRAS, 392, 383

Jones, C. E., Tycner, C., Sigut, T. A. A., Benson, J.A. et al. 2008, ApJ, 687, 598

Kanaan, S., Meilland, A., Stee, P., Zorec, J. et al. 2008, A $₫ A, 486,785$

Kervella, P. \& Domiciano de Souza, A. 2006, A\&A, 453, 1059

Kervella, P. \& Domiciano de Souza, A. 2007, A\&A, 474, L49

Lenorzer, A., de Koter, A. \& Waters, L. B. F. M. 2002, $A \mathscr{\Im} A, 386$, L5

Mackay, F. E., Elias, N. M., Jones, C. E. \& Sigut, T. A. A. 2009, ApJ, 704, 591

Martayan, C., Baade, D. \& Fabregat, J. 2010, A\& A, 509, A11

McSwain, M. V., Huang, W., Gies, D. R., Grundstrom, E. D. et al. 2008, ApJ, 672, 590

McSwain, M. V., Huang, W. \& Gies, D. R. 2009, ApJ, 700, 1216

Meilland, A., Stee, P., Zorec, J. \& Kanaan, S. 2006, A\& $A$, 455, 953

Meilland, A., Stee, P., Vannier, M., Millour, F. et al. 2007a, A\& $A, 464,59$

Meilland, A., Millour, F., Stee, P., Domiciano de Souza, A. et al. 2007b, A\& A, 464, 73

Meilland, A., Millour, F., Stee, P., Spang, A. et al. 2008, A\& $A$, 488, L67

Meilland, A., Stee, P., Chesneau, O. \& Jones, C. 2009, A\&A, 505, 687

Mennickent, R. E., Sabogal, B., Granada, A. \& Cidale, L. 2009, PASP, 121, 125

Millour, F., Meilland, A., Chesneau, O. et al. 2011, A\&A, 526A, 107

Okazaki, A. T. 1996, PASJ, 48, 305

Okazaki, A. T. 1997, A\&A A, 318, 548

Okazaki, A. T. 2007, in: A. T. Okazaki, S. P. Owocki, \& S. Stefl (eds.), Active OB-Stars: Laboratories for Stellar and Circumstellar Physics, ASP-CS 361, p. 230

Oudmaijer, R. D., Parr, A. M., Baines, D. \& Porter, J. M. 2008, A\&A A, 489, 627

Rivinius, T., Baade, D., Stefl, S., Stahl, O. et al. 1998, A\&SA, 333, 125

Schaefer, G. H., Gies, D. R., Monnier, J. D., Richardson, N. et al. 2010, in: T. Rivinius \& M. Curé (eds.), The Interferometric View on Hot Stars, Rev. Mexicana AyA Conference Series 38 , p. 107

Stee, P. \& Meilland, A. 2009, in: J.-P. Rozelot \& C. Neiner (eds.), The Rotation of Sun and Stars, Lecture Notes in Physics 765 (Berlin Springer Verlag), p. 195

Štefl, S., Rivinius, T., Carciofi, A. C., Le Bouquin, J.-B. et al. 2009, A\&GA, 504, 929

Stefl, S., Rivinius, T., Le Bouquin, J.-B., Carciofi, A. et al. 2010, in: T. Rivinius \& M. Curé (eds.), The Interferometric View on Hot Stars, Rev. Mexicana AyA Conference Series 38, p. 89

Tatulli, E., Malbet, F., Ménard, F., Gil, C. et al. 2008, A\& $A, 489,1151$

Touhami, Y., Richardson, N. D., Gies, D. R., Schaefer, G. H. et al. 2010, PASP, 122, 379

Tycner, C., Gilbreath, G. C., Zavala, R. T., Armstrong, J. T. et al. 2006, AJ, 131, 2710

Tycner, C., Jones, C. E., Sigut, T. A. A., Schmitt, H. R. et al. 2008, ApJ, 689, 461

Waters, L. B. F. M. 1986, A\&\&A, 162, 121

Waters, L. B. F., Marlborough, J. M., van der Veen, W. E. C., Taylor, A. R. et al. 1991, A\&A, 244, 120

Wisniewski, J. P., Kowalski, A. F., Bjorkman, K. S., Bjorkman, J. E. et al. 2007, ApJ (Letters), $656, \mathrm{~L} 21$

Wisniewski, J. P., Draper, Z. H., Bjorkman, K. S., Meade, M. R. et al. 2010, ApJ, 709, 1306

Zorec, J., Arias, M. L., Cidale, L. \& Ringuelet, A. E. 2007, A\& A, 470, 239 